\title{
Minimum-Time System-Inversion-Based Motion Planning for Residual Vibration Reduction
}

\author{
Aurelio Piazzi, Member, IEEE, and Antonio Visioli, Member, IEEE
}

\begin{abstract}
In this paper, we present a novel approach, based on system inversion, for the point-to-point motion planning of vibratory servosystems. The idea is to define a suitable parameterized motion law of the load which assures that no oscillations occurs during and at the end of the motion; then, by means of a noncausal system inversion, the command function of the system is determined with a continuous derivative of an arbitrary order. A procedure that minimizes the duration of the movement, taking into account actuator constraints, can then be performed. Comparisons with the well-known input shaping techniques have been performed via both a simulation example and an experimental setup. The proposed method, which is inherently robust to modeling errors, emerges as a very flexible and competitive technique.
\end{abstract}

Index Terms-Open-loop control, system inversion, time optimization, vibratory systems.

\section{INTRODUCTION}

$\mathbf{I}$ T IS WELL KNOWN that the performances of positioning servosystems are limited by the presence of elasticity in the transmissions that introduce vibrations. This generally results in the increasing of the working cycle time in order for the oscillation to vanish after the point-to-point motion has been accomplished. To eliminate such an effect of residual vibrations, two strategies can be implemented: the closed-loop feedback control, which is based on the instantaneous knowledge of the system state, and the open-loop control which consists of an adequate shaping of the command input and requires the knowledge of the system model. In the latter case, a further closed-loop system is generally employed to cope with the effect of disturbances and parameter variations. Many solutions have been proposed in the literature addressing this motion planning aspect. From a theoretical viewpoint, linear quadratic optimal techniques can be adopted to obtain an optimal final-state control, as shown, for example, in [1]; see, also, [2, pp. 127-134]. With another standpoint, Aspinwall has devised a pulse-shaping technique for the forcing function, based on a short, finite Fourier series expression whose coefficient is selected to depress the envelope of the residual response spectrum in desired regions [3]. Other methods proposed by Meckl and Seering consist of using a multiswitch bang-bang forcing function, which gives time-optimal performance [4],

Manuscript received June 8, 1998; revised January 14, 1999 and July 15, 1999. Recommended by Technical Editor K. Ohnishi. This work was supported in part by MURST scientific research funds and by ASI (Italian Space Agency).

A. Piazzi is with the Dipartimento di Ingegneria dell'Informazione, University of Parma, I-43100 Parma, Italy (e-mail: aurelio@ce.unipr.it).

A. Visioli is with the Dipartimento di Elettronica per l'Automazione, University of Brescia, I-25123 Brescia, Italy (e-mail: visioli@ing.unibs.it).

Publisher Item Identifier S 1083-4435(00)01307-7. or adding up harmonics of ramped sinusoid functions in order to approximate as close as possible a bang-bang function, but minimizing the energy introduced at system resonance frequencies [5]. The most well-known technique is, however, the input shaping, which has been developed in the last decade [6]-[11]. It consists of convolving a sequence of impulses, also known as the input shaper, with a desired system command to generate the system command that is then actually used to drive the system. Different impulse sequences (which depend on the natural frequencies and damping of the system to be controlled) can be employed in order to cope with multiple modes [7], [8], [11] and to increase robustness. In particular, if a single oscillation mode is addressed, the zero-vibration (ZV), zero-vibration and zero-derivative (ZVD), and ZVDD (also the second derivative is set to zero) shapers aim to force to zero the residual vibration if the real system corresponds to the nominal one [6]. An alternative procedure uses extra-insensitive (EI) constraints to increase the width of the notch of the insensitivity curve, allowing a small residual vibration at the modeling frequency [9]. Subsequent developments of this approach are the two-humped and three-humped EI [10]. In general, the increasing of robustness of the system (obtained with a larger number of impulses adopted in the shaper) is paid with an increasing of system delays, due to the convolution process.

In this paper, we present a novel approach to solve the problem of residual vibration, which can be briefly described as follows. An arbitrarily smooth closed-form motion law for the load of the system is determined in such a way that guarantees the absence of oscillations during and at the end of the motion. The function is parameterized by the time interval $\tau$ of the point-to-point motion. Then, by means of a noncausal system inversion, the corresponding parameterized actuator input is determined. Finally, a time optimization is performed in order to minimize the motion time taking into account actuator constraints. Polynomial functions are suitable to be adopted as output functions for this method, since monotonicity can be easily obtained and they assure the smoothness of the input function and its derivatives until an arbitrarily prefixed order. System invertibility has been the subject of numerous investigations since the 1960's, especially for linear multi-input multi-output (MIMO) systems, for which issues such as test conditions for system invertibility, algorithmic constructions of the inverse system, and stability of the inverse system were addressed [12]-[15]. Another issue is left-invertibility or right-invertibility of a system. The latter concept is also known with the more illuminating term of functional reproducibility, which indicates the possibility to reproduce a given output function by a suitable input function [16], [17]. In recent 


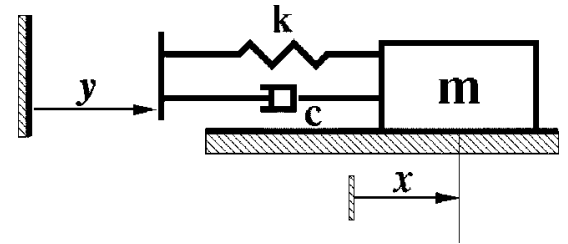

Fig. 1. Model of system with elastic linkage.

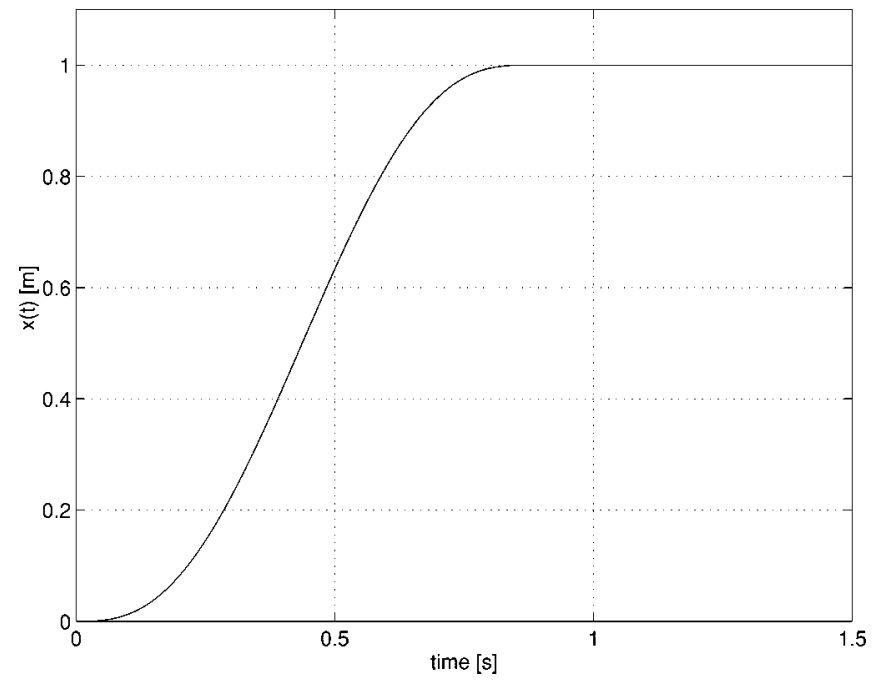

Fig. 2. Output function of the system (load motion).

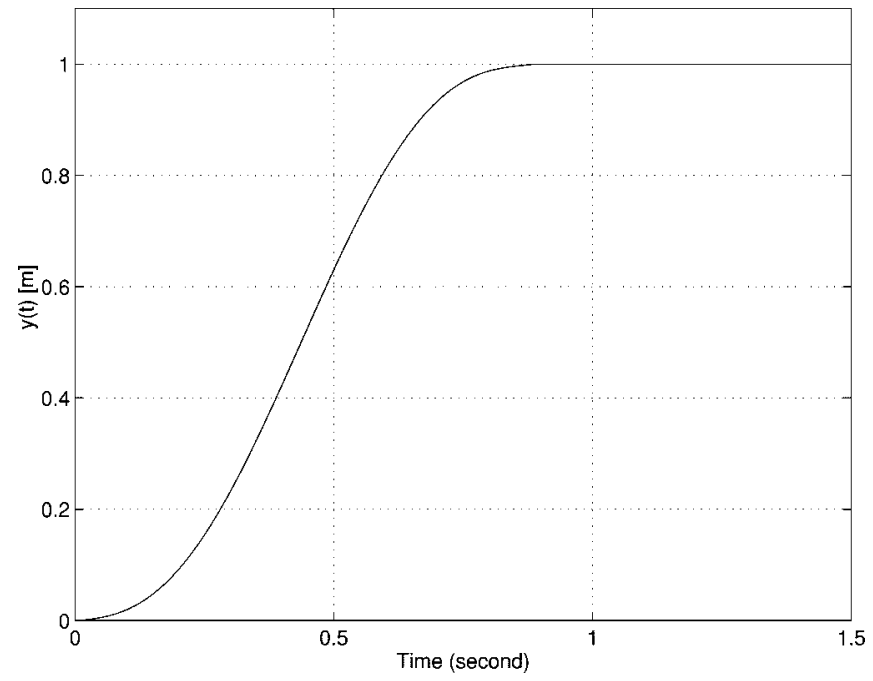

Fig. 3. Input function of the system (position command signal).

years, the system inversion idea has been applied to perfect or quasi-perfect tracking for linear and nonlinear dynamic systems [18]-[21]. In these papers, the main emphasis is on the construction of noncausal stable inverses in the presence of unstable zero dynamics. For our purpose, the functional reproducibility property necessary to perform a stable noncausal inversion on the output motion is always secured because the relevant system is scalar and minimum phase.

The paper is organized as follows. In Section II, the open-loop control strategy based on system inversion is proposed. The procedure to minimize the motion time subject to actuator constraints is demonstrated in Section III. Sections IV and V show

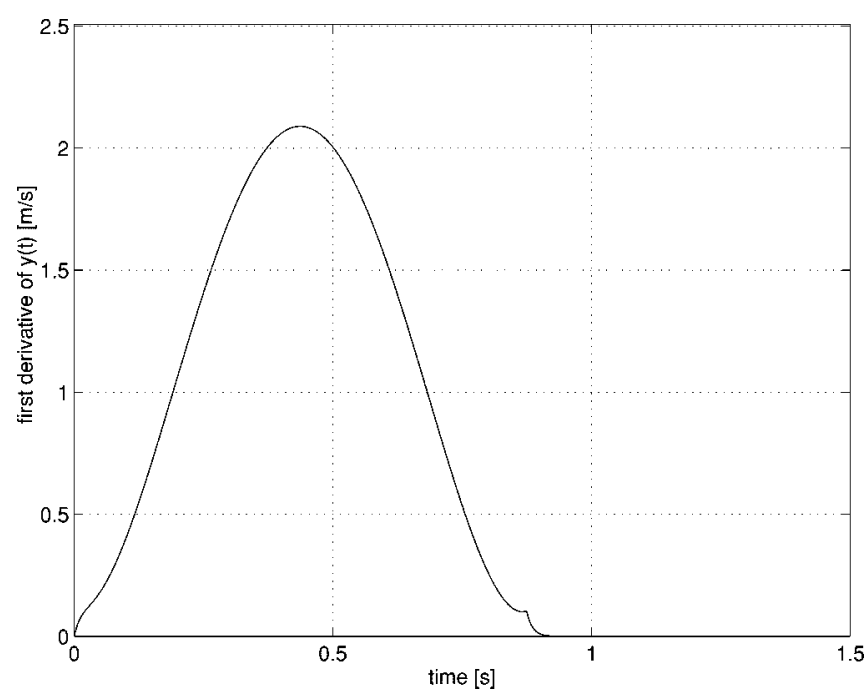

Fig. 4. First derivative of the input function of the system (velocity command signal).

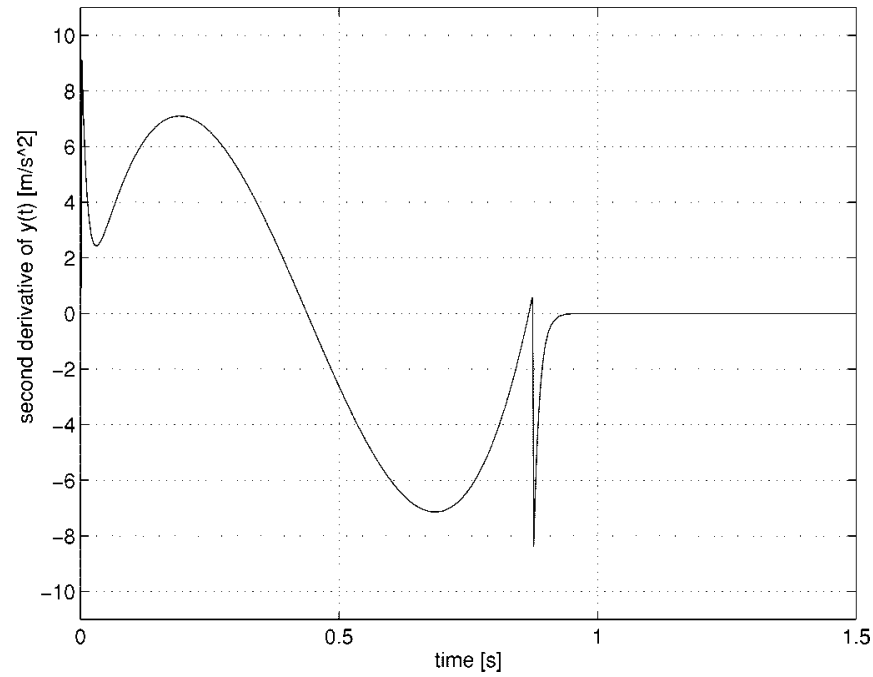

Fig. 5. Second derivative of the input function of the system (acceleration command signal).

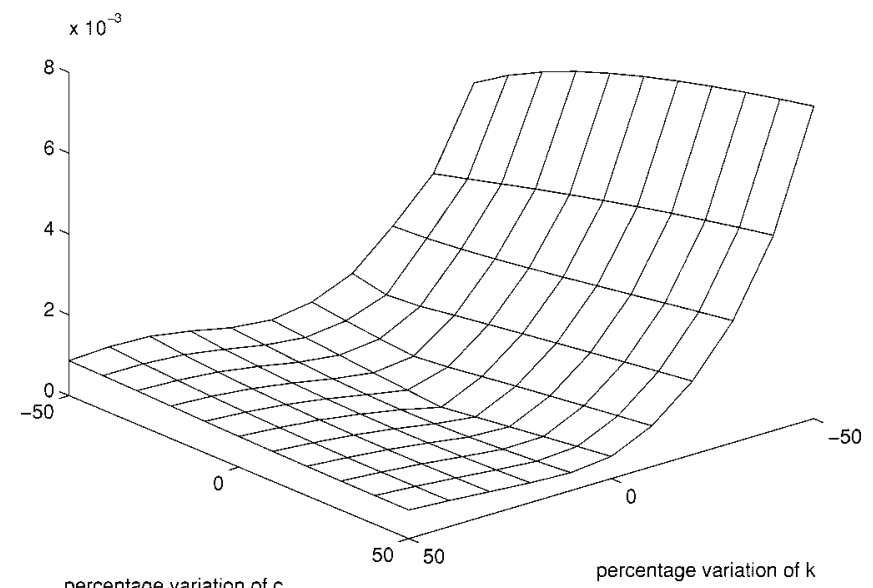

Fig. 6. Maximum amplitude of residual vibration for different values of model parameters.

simulation results for a given example. The former section applies the system inversion motion planning and presents the re- 


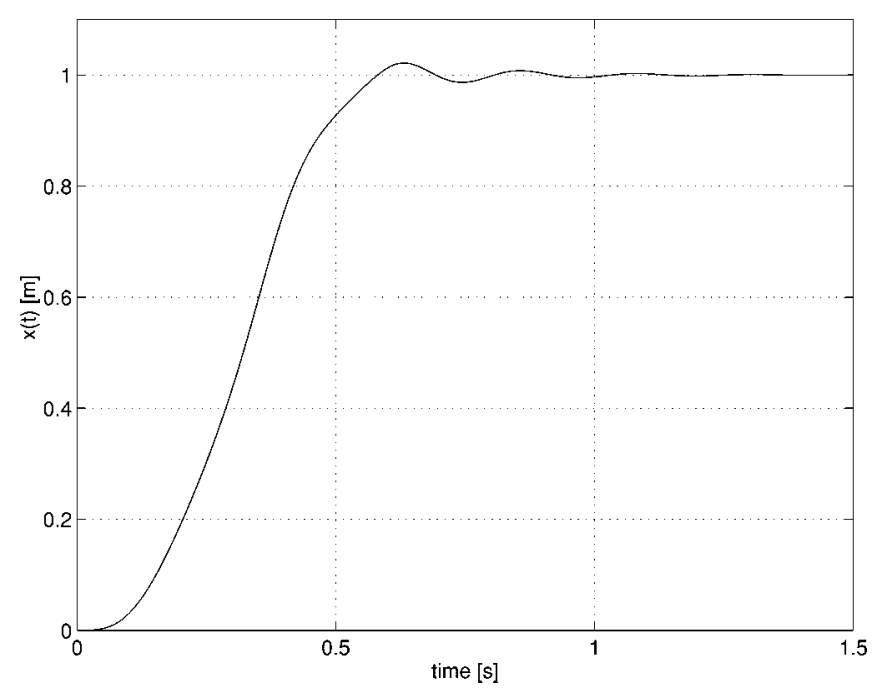

Fig. 7. Motion of the load using a bang-bang function of amplitude $y_{\max }^{(2)}=$ 10 (bang-bang I case).

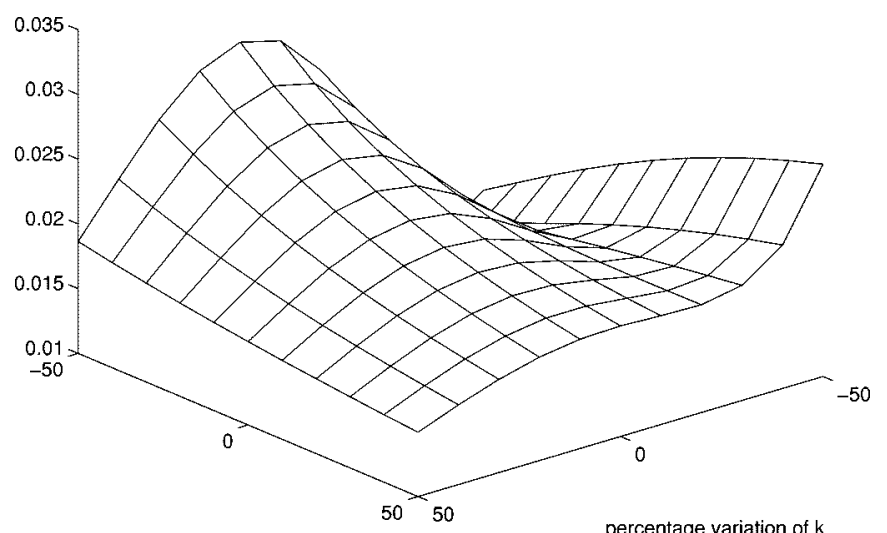

percentage variation of $c$

percentage variation of $k$

Fig. 8. Maximum amplitude of residual vibration using a bang-bang acceleration input function of amplitude $y_{\max }^{(2)}=10$ (bang-bang I case).

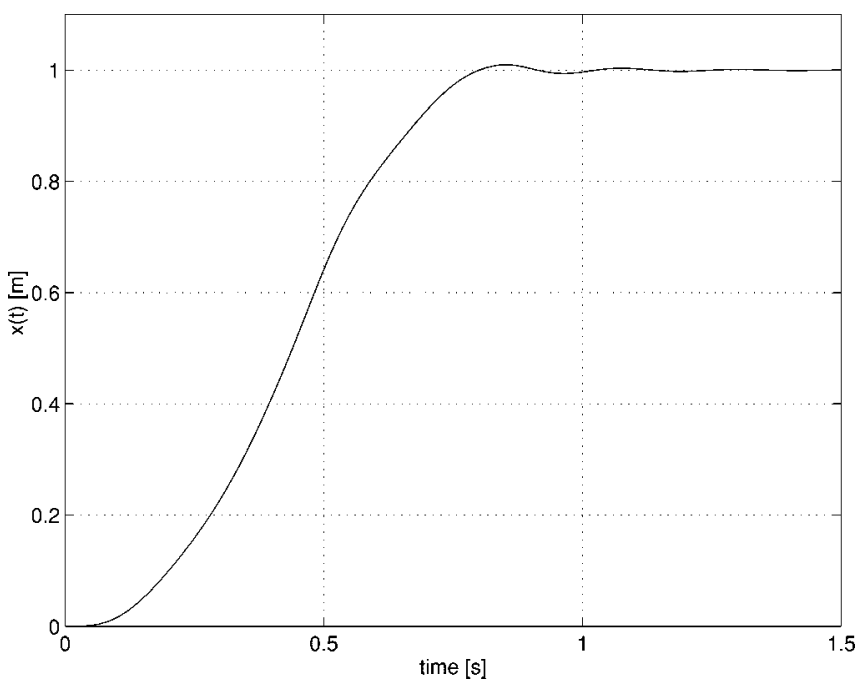

Fig. 9. Motion of the load using a bang-bang function of duration $\tau^{*}=0.874$ s (bang-bang II case).

lated robustness analysis, whereas the latter one is devoted to comparisons with the input shaping techniques. Experimental results with comparisons for a linear flexible joint (by Quanser

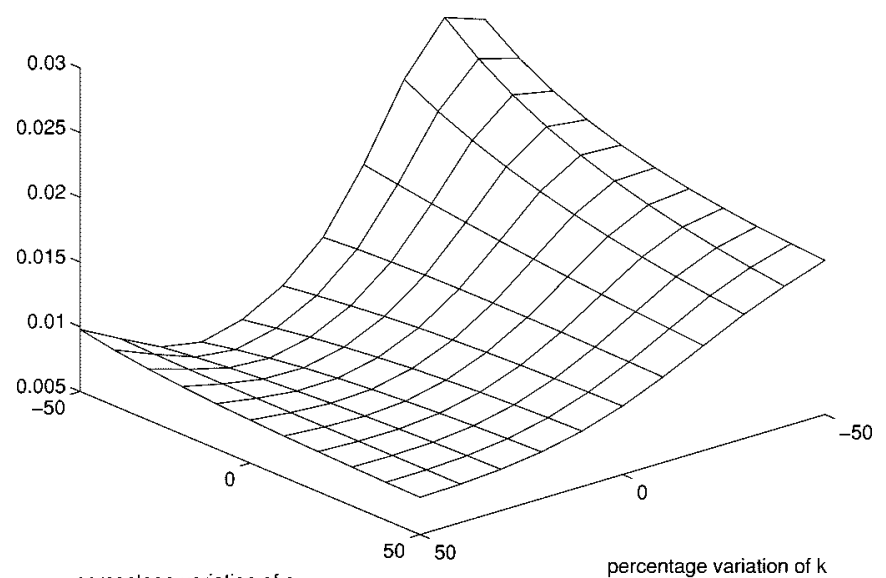

percentage variation of $c$

Fig. 10. Maximum amplitude of residual vibration using a bang-bang acceleration input function of duration $\tau^{*}=0.874 \mathrm{~s}$ (bang-bang II case).

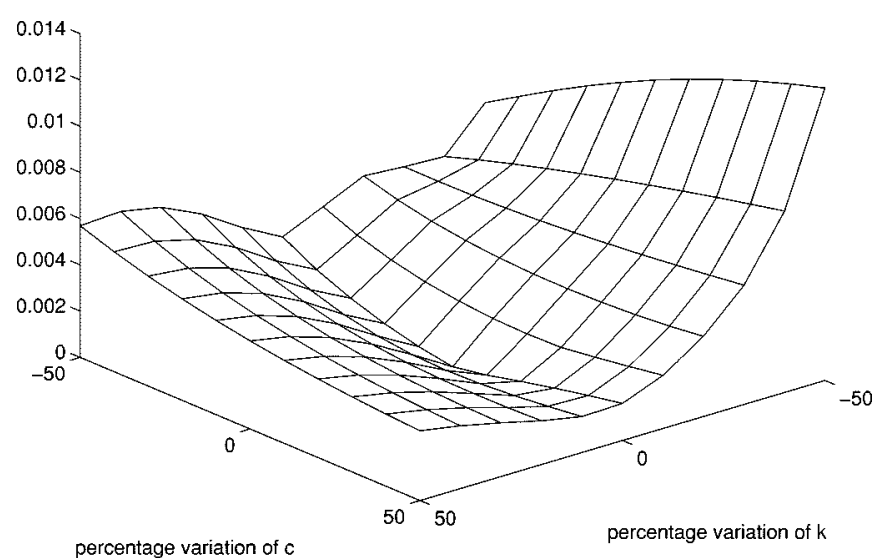

Fig. 11. Maximum amplitude of residual vibration using the $\mathrm{ZV}$ impulse shaper method for different values of the model parameter.

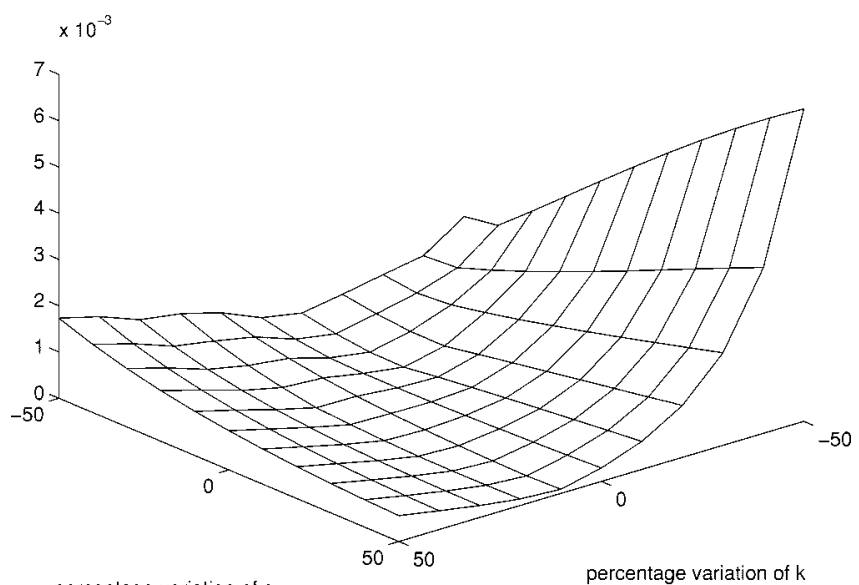

percentage variation of $\mathrm{c}$

Fig. 12. Maximum amplitude of residual vibration using the ZVD impulse shaper method for different values of the model parameter.

Consulting) are reported in Section VI. Conclusions are drawn in Section VII.

\section{System-INVERSION-BASED Motion PlanNing}

Many mechanical positioning servosystems (e.g., robot arms or overhead cranes) have an elastic transmission, mainly intro- 
duced by the gearbox [22], which can be simply described by the model shown in Fig. 1 where $y$ is the coordinate representing the motor shaft displacement, $x$ is the coordinate representing the mass displacement, $m$ is the load mass, $k$ the stiffness constant, and $c$ the damping of the transmission [23]. The well-known linear relation which links $x$ and $y$ has the following differential form:

$$
m \ddot{x}+c \dot{x}+k x=c \dot{y}+k y
$$

which can be rewritten as

$$
\ddot{z}+2 \xi \omega_{n} \dot{z}+\omega_{n}^{2} z=-\ddot{y}
$$

where $z=x-y, \omega_{n}=\sqrt{k / m} \mathrm{rad} \cdot \mathrm{s}^{-1}$ is the frequency of the oscillatory mode, and $\xi=c / 2 m \omega_{n}$ is the damping ratio. In general (we refer, for example, to the motion planning of manipulators), the elasticity of the transmission is not taken into account and the motion is planned on $y$ [the input of the system (1)] hoping that the motion of $m$ is very close to it. Limits of the actuators have to be considered in the choice of the motion law of $y$ so that it can be followed in practical cases. Vibrations are generally neglected in this phase and, in case they are not tolerable, actuator exertion has to be reduced. This is, however, a severe drawback for high-performance systems. The approach presented in this paper shows how it is possible to overcome this limitation by calculating the motion of $y$ in order to get a predefined motion of $m$, assuming to have the knowledge of the values of the parameters of the system. The idea is to define a priori and impose the desired function $x(t)$ and then use dynamic inversion in order to obtain $y(t)$ that is the input movement of the motor shaft that causes the desired output $x(t)$. The choice of the function $x(t)$ assumes great importance for the whole procedure. Polynomial functions present important properties, illustrated in the following, that make them particularly suitable for the purpose described above. The general expression of a polynomial function is

$$
x(t)=a_{n} t^{n}+a_{n-1} t^{n-1}+\cdots+a_{1} t+a_{0} .
$$

The choice of $n$, the degree of the polynomial, results from the following property, regarding the differentiability of the input of the system. Denote by $C^{(h)}$ the class of functions which have an $h$ th continuous derivative. Let also $y(t)=0$ and $x(t)=0$ for all $t<0$ (at time $t=0^{-}$all initial conditions are equal to zero).

Property 1: Assume that $h \geq 1$. For the system described by the differential equation (1), if $x(t) \in C^{(h)}$, then it is functional reproducible by a unique $y(t) \in C^{(h-1)}$.

Proof: Denote by $G(s)$ the transfer function of the system (1)

$$
G(s)=\frac{c s+k}{m s^{2}+c s+k} .
$$

We can write $G^{-1}(s)$, dividing the denominator by the numerator, as

$$
G^{-1}(s)=\gamma_{1} s+\gamma_{0}+\frac{\alpha_{0}}{\beta_{1} s+1}
$$

where

$$
\gamma_{1}=m / c \quad \gamma_{0}=1-m k / c^{2} \quad \alpha_{0}=m k / c^{2} \quad \beta_{1}=c / k .
$$

Applying the Laplace transform also to $x(t)$ and $y(t)$, it follows, trivially

$$
\begin{aligned}
Y(s) & =G^{-1}(s) X(s) \\
& =\gamma_{1} s X(s)+\gamma_{0} X(s)+\frac{\alpha_{0}}{\beta_{1} s+1} X(s) .
\end{aligned}
$$

In the time domain, the expression (5) results ( $v$ is the real variable of the integrand function)

$$
y(t)=\gamma_{1} \dot{x}(t)+\gamma_{0} x(t)+\int_{0}^{t} g_{0}(t-v) x(v) d v
$$

where, denoting by $\mathcal{L}^{-1}$ the inverse Laplace transform operator

$$
g_{0}(t)=\mathcal{L}^{-1}\left[\frac{\alpha_{0}}{\beta_{1} s+1}\right] .
$$

Now, multiplying by $s$ both the right and the left part of (5), we obtain

$$
s Y(s)=\gamma_{1} s^{2} X(s)+\gamma_{0} s X(s)+\frac{\alpha_{0}}{\beta_{1}} X(s)+\frac{\delta_{0}}{\beta_{1} s+1} X(s)
$$

where $\delta_{0}$ is the appropriate coefficient. Multiplying again by $s$

$$
\begin{aligned}
s^{2} Y(s)=\gamma_{1} s^{3} X(s)+\gamma_{0} s^{2} X(s) & +\frac{\alpha_{0}}{\beta_{1}} s X(s) \\
& +\frac{\delta_{0}}{\beta_{1}} s X(s)+\frac{\delta_{1}}{\beta_{1} s+1} X(s) .
\end{aligned}
$$

Generalizing, multiplying $n$ times by $s$, we have

$$
\begin{array}{r}
s^{n} Y(s)=\gamma_{1} s^{n+1} X(s)+\gamma_{0} s^{n} X(s)+\frac{\alpha_{0}}{\beta_{1}} s^{n-1} X(s) \\
+\cdots+\frac{\delta_{n-2}}{\beta_{1}} X(s)+\frac{\delta_{n-1}}{\beta_{1} s+1} X(s) .
\end{array}
$$

Applying to (8) the Laplace inverse transform, it results in

$$
\begin{aligned}
y^{(n)}(t)=\gamma_{1} x^{(n+1)}(t)+\gamma_{0} x^{(n)}(t)+\frac{\alpha_{0}}{\beta_{1}} x^{(n-1)}(t) \\
\\
+\cdots+\frac{\delta_{n-2}}{\beta_{1}} x(t)+\int_{0}^{t} g_{n}(t-v) x(v) d v
\end{aligned}
$$

where

$$
g_{n}(t)=\mathcal{L}^{-1}\left[\frac{\delta_{n-1}}{\beta_{1} s+1}\right] .
$$

From (9), it follows that, if $x(t) \in C^{(n+1)}$, then $y^{(n)}(t)$ is a continuous function because all the functions in the right side of (9) are continuous. Hence, $y(t) \in C^{(n)}$, so if $n=h-1$, the property is proved.

The point-to-point motion of the load from position zero to position $q$ has to be completed in the time interval $[0, \tau]$ with $\tau$ being the free parameter that defines the motion duration time. Therefore, formally, we have $x(t ; \tau)=0$ if $t \leq 0$ and $x(t ; \tau)=$ $q$ if $t \geq \tau$. The function $x(t ; \tau)$ over $[0, \tau]$ is chosen to be a $(2 h+$ 1)-degree polynomial in order to assure $x(t ; \tau) \in C^{(h)}$ over $(-\infty,+\infty)$. The function class $C^{(h)}$ is selected, according to 


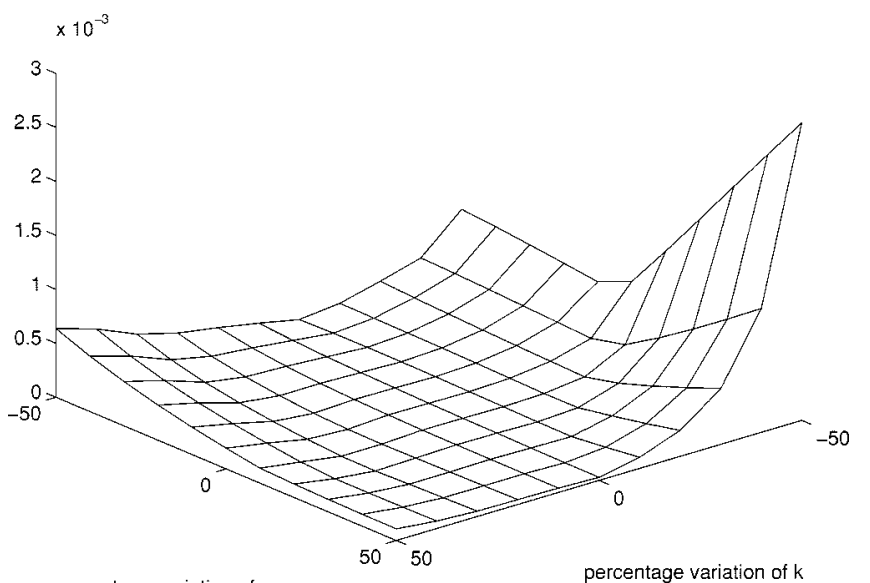

percentage variation of $\mathrm{c}$

Fig. 13. Maximum amplitude of residual vibration using the ZVDD impulse shaper method for different values of the model parameter.

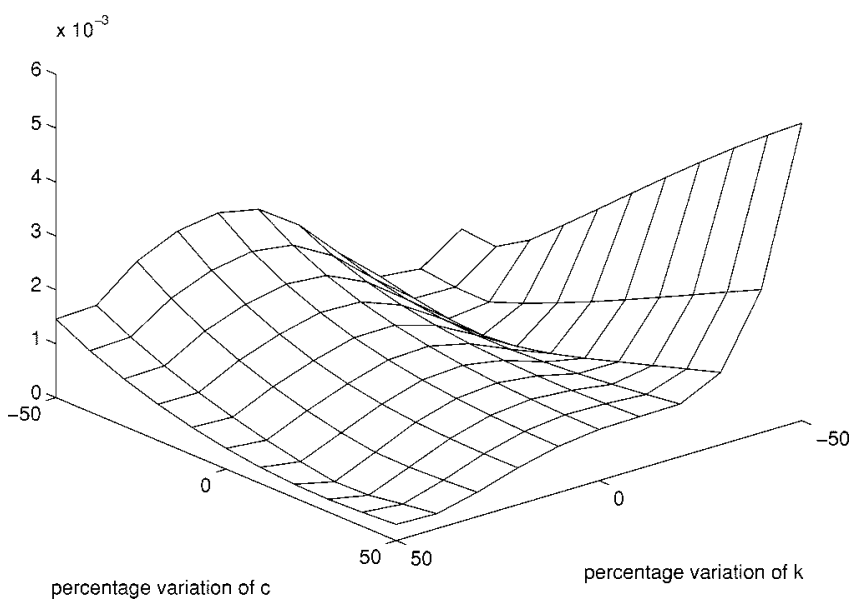

Fig. 14. Maximum amplitude of residual vibration using the EI impulse shaper method for different values of the model parameter.

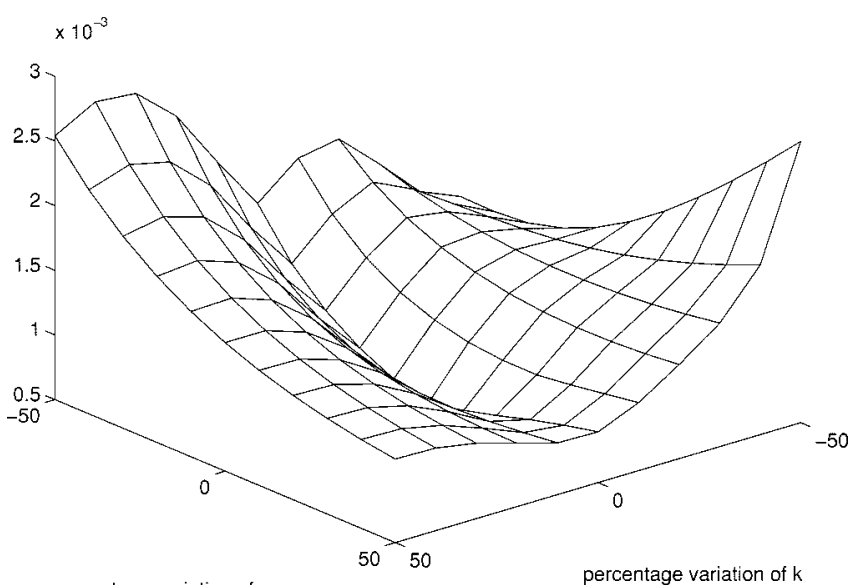

percentage variation of $c$

percentage variation of $k$

Fig. 15. Maximum amplitude of residual vibration using the two-humped EI impulse shaper method for different values of the model parameter.

Property 1 , such as $y(t ; \tau)$, the corresponding input that causes the planned motion $x(t ; \tau)$, has $h-1$ continuous derivatives.

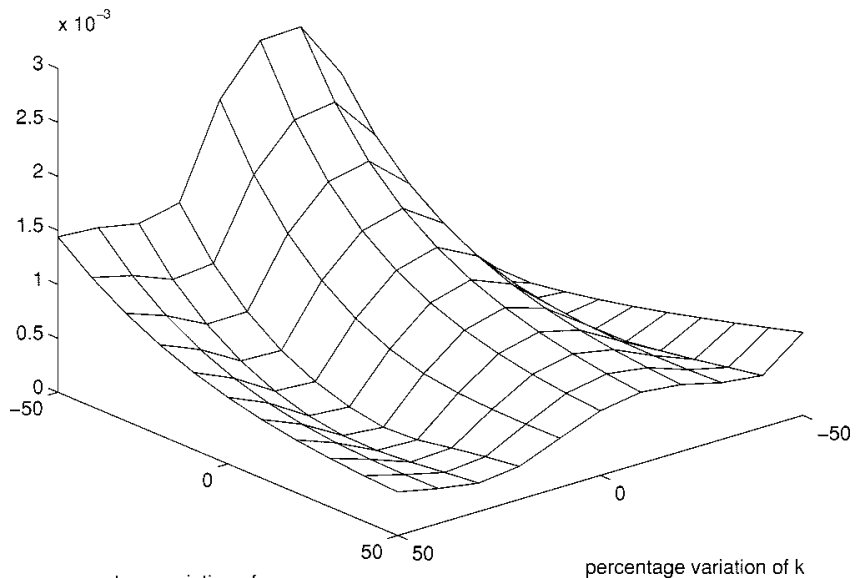

percentage variation of $\mathrm{C}$

Fig. 16. Maximum amplitude of residual vibration using the three-humped EI impulse shaper method for different values of the model parameter.

The values of the $2 h+2$ coefficients of the sought polynomial can be found by solving the following linear algebraic system:

$$
\left\{\begin{array}{cc}
x(0 ; \tau)=0, & x(\tau ; \tau)=q \\
x^{(1)}(0 ; \tau)=0, & x^{(1)}(\tau ; \tau)=0 \\
\vdots & \vdots \\
x^{(h)}(0 ; \tau)=0, & x^{(h)}(\tau ; \tau)=0 .
\end{array}\right.
$$

The general closed-form expression of polynomial $x(t ; \tau)$ with $t \in[0, \tau]$ that results from (10) is given by the following remarkable formula:

$$
x(t ; \tau)=\alpha(h, \tau) q \int_{0}^{t} v^{h}(\tau-v)^{h} d v
$$

with positive coefficient $\alpha(h, \tau)=\left(\int_{0}^{\tau} v^{h}(\tau-v)^{h} d v\right)^{-1}$. The above formula (11) can be easily proven taking into account that

$$
\dot{x}(t ; \tau)=\alpha(h, \tau) q t^{h}(\tau-t)^{h}, \quad t \in[0, \tau] .
$$

From (12), it is also clear that $x(t ; \tau)$ is monotonically increasing, since $\dot{x}(t ; \tau)$ is positive all over $(0, \tau)$; in this way, it is guaranteed that there are no oscillations during the whole motion of $m$.

Having synthesized, according to (11), the desired parameterized load motion $x(t ; \tau)$ as a $C^{(h)}$-class function over $(-\infty,+\infty)$, we proceed to determine, by system inversion, the (unique) corresponding input function $y(t ; \tau)$ that belongs to $C^{(h-1)}$ over $(-\infty,+\infty)$ (see Property 1 ). By virtue of the explicit inversion formula (6) and using the definitions appearing in (4) and (7), after a few passages, the following closed-form expression of $y(t ; \tau)$ for $t \geq 0$ can be derived (obviously, $y(t ; \tau)=0$ if $t<0$ ):

$$
\begin{array}{r}
y(t ; \tau)=\frac{m}{c} \dot{x}(t ; \tau)+\left(1-\frac{m k}{c^{2}}\right) x(t ; \tau)+\frac{m k^{2}}{c^{3}} e^{-(k / c) t} \\
\cdot \int_{0}^{t} e^{(k / c) v} x(v ; \tau) d v
\end{array}
$$


TABLE I

COMPARISON OF THE DifFERENT METHODOLOGIES FOR THE SimUlation EXMAPLE

\begin{tabular}{l|c|c|c}
\hline Methodology & \multirow{2}{*}{$\begin{array}{c}\text { Scheduled } \\
\text { motion time }[\mathrm{s}]\end{array}$} & \multicolumn{2}{|c}{ Max. amplitude of residual vibration [mm] } \\
\cline { 3 - 4 } & 0.874 & 0 & nominal case \\
\hline System inversion & 0.632 & 21.7 & 7.7 \\
\hline Bang-bang I & 0.874 & 9.9 & 30.3 \\
\hline Bang-bang II & 0.745 & 0 & 25.6 \\
\hline ZV shaper & 0.857 & 0 & 12.7 \\
\hline ZVD shaper & 0.970 & 0 & 6.8 \\
\hline ZVDD shaper & 0.857 & 1.2 & 2.8 \\
\hline EI shaper & 0.962 & 0 & 5.5 \\
\hline Two-humped EI shaper & 1.075 & 0.9 & 2.7 \\
\hline Three-humped EI shaper & & & 2.7 \\
\hline
\end{tabular}

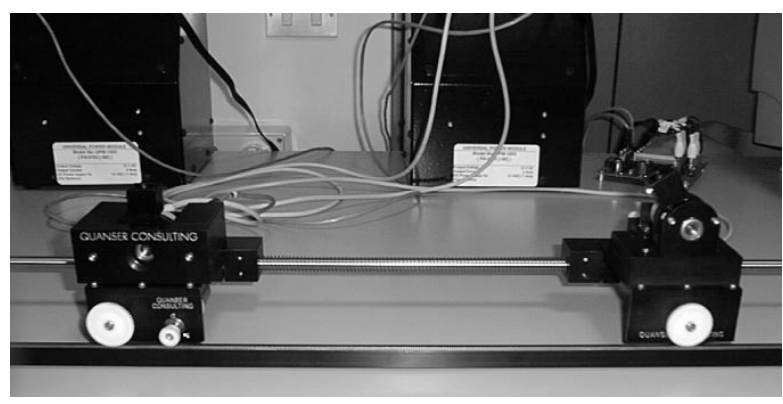

Fig. 17. The experimental setup (made by Quanser Consulting) adopted in the experiments.

This expression can be simplified for $t \geq \tau$. Indeed, consider that $x(t ; \tau)=q$ and $\dot{x}(t ; \tau)=0$ for $t \geq \tau$. Hence,

$$
\begin{aligned}
& y(t ; \tau)=\left(1-\frac{m k}{c^{2}}\right) q+\frac{m k^{2}}{c^{3}} e^{-(k / c) t} \\
& \cdot\left[\int_{0}^{\tau} e^{(k / c) v} x(v ; \tau) d v+\int_{\tau}^{t} e^{(k / c) v} q d v\right] .
\end{aligned}
$$

Explicitly computing the second integral appearing in the above expression, we obtain

$$
\begin{array}{rl}
y(t ; \tau)=q+\frac{m k^{2}}{c^{3}} e^{-(k / c) t} \int_{0}^{\tau} e^{(k / c) v} & x(v ; \tau) d v \\
& -q \frac{m k}{c^{2}} e^{-(k / c)(t-\tau)} .
\end{array}
$$

Taking into account that

$$
\begin{aligned}
& y(\tau ; \tau)=\left(1-\frac{m k}{c^{2}}\right) q+\frac{m k^{2}}{c^{3}} e^{-(k / c) \tau} \\
& \cdot \int_{0}^{\tau} e^{(k / c) v} x(v ; \tau) d v
\end{aligned}
$$

it follows that

$$
\begin{aligned}
& \frac{m k^{2}}{c^{3}} e^{-(k / c) t} \int_{0}^{\tau} e^{(k / c) v} x(v ; \tau) d v \\
& =y(\tau ; \tau) e^{-(k / c)(t-\tau)}-\left(1-\frac{m k}{c^{2}}\right) q e^{-(k / c)(t-\tau)} .
\end{aligned}
$$

By substitution, we eventually obtain

$$
y(t ; \tau)=q+[y(\tau, \tau)-q] e^{-(k / c)(t-\tau)}, \quad t \geq \tau
$$

From (14), it is apparent that $y(t ; \tau)$ is all over bounded because the excited zero mode $e^{-(k / c) t}$ is stable.

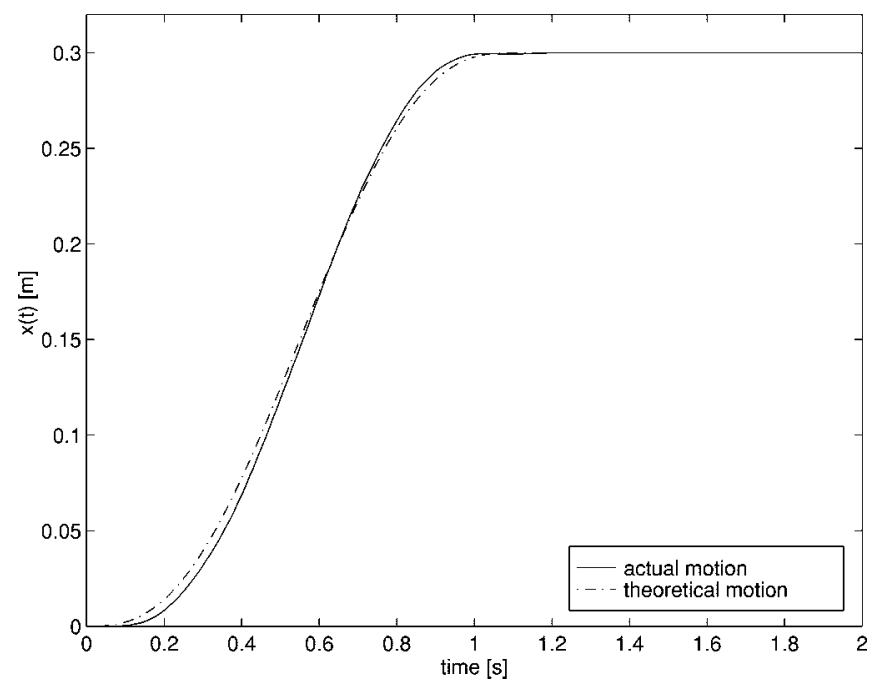

Fig. 18. Theoretical and actual load motion obtained with the system-inversion-based methodology.

\section{TIME OPTIMIZATION}

Once parameter $h$ has been fixed in order to choose the class of the motion law $\left(y(t ; \tau) \in C^{(h-1)}\right)$, the other parameter $\tau$, the duration of the output motion, can be selected to satisfy actuator constraints. These constraints can be introduced, for example, as bounds on the absolute values of the input $y(t ; \tau)$ and its derivatives until a prefixed order $l(l \leq h)$. Hence, the following semi-infinite optimization problem naturally arises (where $y_{\max }^{(i)}$ denotes the given bound on the $i$ th-order derivative):

$\operatorname{minimize} \tau$

subject to

$$
\left|y^{(i)}(t ; \tau)\right| \leq y_{\max }^{(i)}, \quad \forall t \in[0, \tau], \quad i=0,1, \cdots, l .
$$

The optimal solution $\tau^{*}$ of the above problem can be found by means of the following algorithm, which is a typical bisection algorithm.

\footnotetext{
1. Set $\tau_{\min }=0$.

2. Determine an initial value for $\tau_{\max }$ such as
}

$$
\max _{t \in\left[0, \tau_{\max }\right]}\left|y^{(i)}\left(t ; \tau_{\max }\right)\right| \leq y_{\max }^{(i)}, \quad i=1, \cdots, l .
$$




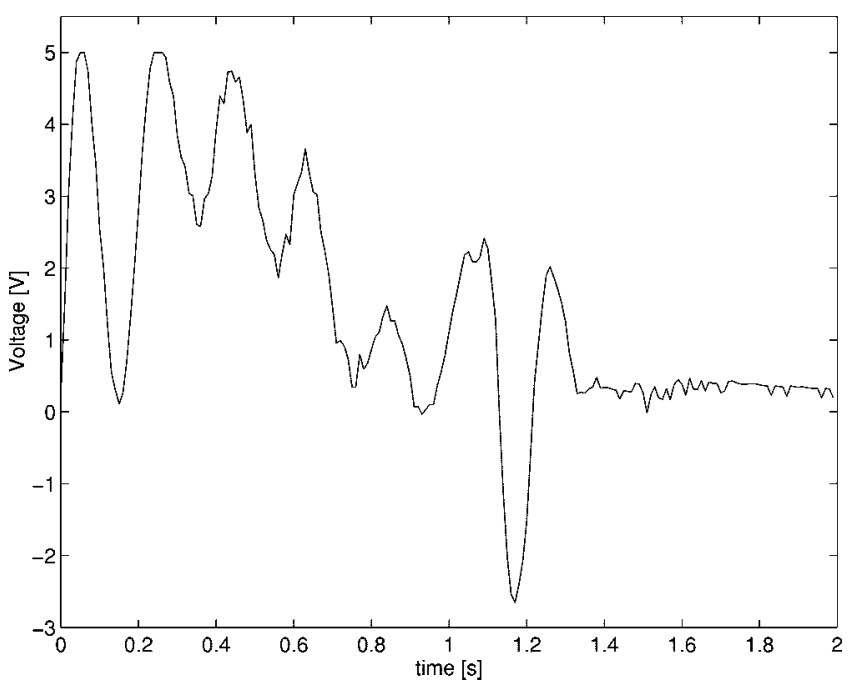

Fig. 19. Motor voltage during the point-to-point motion with the system-inversion-based methodology.

3. Set $\tau=\left(\tau_{\min }+\tau_{\max }\right) / 2$.

4. If $\max _{t \in[0, \tau]}\left|y^{(i)}(t ; \tau)\right| \leq y_{\max }^{(i)}, \quad i=1, \cdots, l$ then set $\tau_{\max }=\tau$ else set $\tau_{\min }=\tau$.

5. If $\left(\tau_{\max }-\tau_{\min }\right)>\varepsilon$ then goto 3 .

6. Set $\tau^{*}=\tau_{\max }$.

7. End.

Remark 1: At step 4, the required computation of the global maximum of $\left|y^{(i)}(t ; \tau)\right|$ over $[0, \tau]$ can be successfully performed by means of an interval algorithm which implements a branch-and-bound strategy with the bounding given by a suitable inclusion function [24], [25].

The initial value of $\tau_{\max }$ can be easily found starting from a reasonable value and, if it does not satisfy the constraints of step 2 , multiplying it repeatedly by a constant $\lambda>1$ until the condition becomes true. The other precision parameter $\varepsilon>0$ determines the terminal condition of the algorithm at point 5 . On the practical side $h \geq 2$ and we can typically impose constraints on $y^{(1)}(t ; \tau), y^{(2)}(t ; \tau)$ which correspond to limits on velocity and acceleration of the actuator.

\section{SIMULATION RESUlTS}

As an illustrative example, we considered the following system:

$$
G(s)=\frac{9 s+800}{s^{2}+9 s+800}
$$

where $m=1 \mathrm{~kg}, k=800 \mathrm{~kg} \cdot \mathrm{s}^{-2}$, and $c=9 \mathrm{~kg} \cdot \mathrm{s}^{-1}$, which corresponds to a natural frequency $\omega=28.28 \mathrm{rad} \cdot \mathrm{s}^{-1}$ and a damping ratio $\xi=0.16$. For the sake of simplicity, we considered a point-to-point motion from 0 to $q=1 \mathrm{~m}$ adopting a polynomial of fifth order as output function $x(t ; \tau)(h=2)$ so that the first derivative (velocity) of the input function $y(t ; \tau)$ is continuous. Hence, we have

$$
x(t ; \tau)=q\left(\frac{6}{\tau^{\tilde{5}}} t^{\tilde{5}}-\frac{15}{\tau^{4}} t^{4}+\frac{10}{\tau^{3}} t^{3}\right) \quad t \in[0, \tau] .
$$

The corresponding input function results, for $t \in[0, \tau]$

$$
\begin{aligned}
y(t ; \tau)=q[ & -360 \frac{c^{2} m}{k^{3} \tau^{4}}-720 \frac{c^{3} m}{k^{4} \tau^{5}}-60 \frac{c m}{k^{2} \tau^{3}} \\
& +\left(360 \frac{c m}{k^{2} \tau^{4}}+720 \frac{c^{2} m}{k^{3} \tau^{5}}+60 \frac{m}{k \tau^{3}}\right) t \\
& +\left(180 \frac{m}{k \tau^{4}}+360 \frac{c m}{k^{2} \tau^{5}}\right) t^{2} \\
& +\left(10 \frac{1}{\tau^{3}}+120 \frac{m}{k \tau^{5}}\right) t^{3}-15 \frac{1}{\tau^{4}} t^{4}+6 \frac{1}{\tau^{5}} t^{5} \\
& \left.+\left(360 \frac{c^{2} m}{k^{3} \tau^{4}}+720 \frac{c^{3} m}{k^{4} \tau^{5}}+60 \frac{c m}{k^{2} \tau^{3}}\right) e^{-(k / c) t}\right] .
\end{aligned}
$$

Optimization has been performed on the motion time $\tau$ taking into account the following constraints on the input function and its derivatives $(l=2)$ :

$$
y_{\max }^{(0)}=2 \mathrm{~m} \quad y_{\max }^{(1)}=5 \mathrm{~m} \cdot \mathrm{s}^{-1} \quad y_{\max }^{(2)}=10 \mathrm{~m} \cdot \mathrm{s}^{-2} .
$$

The resulting optimal motion time $\tau^{*}$ is equal to $0.874 \mathrm{~s}$. Substituting the parameter values in (16), we have for $t \in\left[0, \tau^{*}\right]$

$$
\begin{array}{r}
y\left(t ; \tau^{*}\right)=-0.00136+0.12124 t-0.39553 t^{2}+15.272 t^{3} \\
-25.707 t^{4}+11.765 t^{\tilde{5}}+0.00136 e^{-(800 / 9) t} .
\end{array}
$$

Taking into account (14), for $t>\tau^{*}$ the input function is

$$
y\left(t ; \tau^{*}\right)=1-0.00117 e^{-(800 / 9)(t-0.874)} .
$$

The corresponding output function is shown in Fig. 2, while the input function and its first and second derivatives are plotted, respectively, in Figs. 3-5. It can be noted that the significative bound is the one on the acceleration input signal. A robustness analysis has been done applying the optimal input to the system (15) where the damping $c$ and the stiffness constant $k$ has been perturbed in a range of $\pm 50 \%$ of their nominal values. With a tight gridding over the uncertain parameter domain, the maximum amplitude of the residual vibration (that is the absolute value of the maximum difference between the actual output and the steady-state value after the scheduled motion time) has been plotted in Fig. 6. A worst case vibration amplitude equal to $7.7 \cdot 10^{-3} \mathrm{~m}$ results. As a comparison, the motion of the load for a bang-bang acceleration input signal of amplitude equal to $y_{\max }^{(2)}=10 \mathrm{~m} \cdot \mathrm{s}^{-2}$ is reported in Fig. 7 (bang-bang I case). The corresponding (scheduled) motion time is $0.6325 \mathrm{~s}$. In this case, the resulting maximum amplitude of the residual vibration is $21.7 \cdot 10^{-3} \mathrm{~m}$. A robustness analysis has been performed as above with this input, and the amplitudes of the residual vibration for the different values of the parameters of the systems are shown in Fig. 8. The worst case vibration amplitude amounts to $30.3 \cdot 10^{-3} \mathrm{~m}$. It can be noted that the faster output transient obtained with the bang-bang input is paid with residual vibrations for the nominal and perturbed system. Comparing the robustness analyses shown by Figs. 6 and 8, the better performance of the systems-inversion-based method with respect to the bang-bang acceleration technique is evident. This is also confirmed by applying a bang-bang acceleration function 


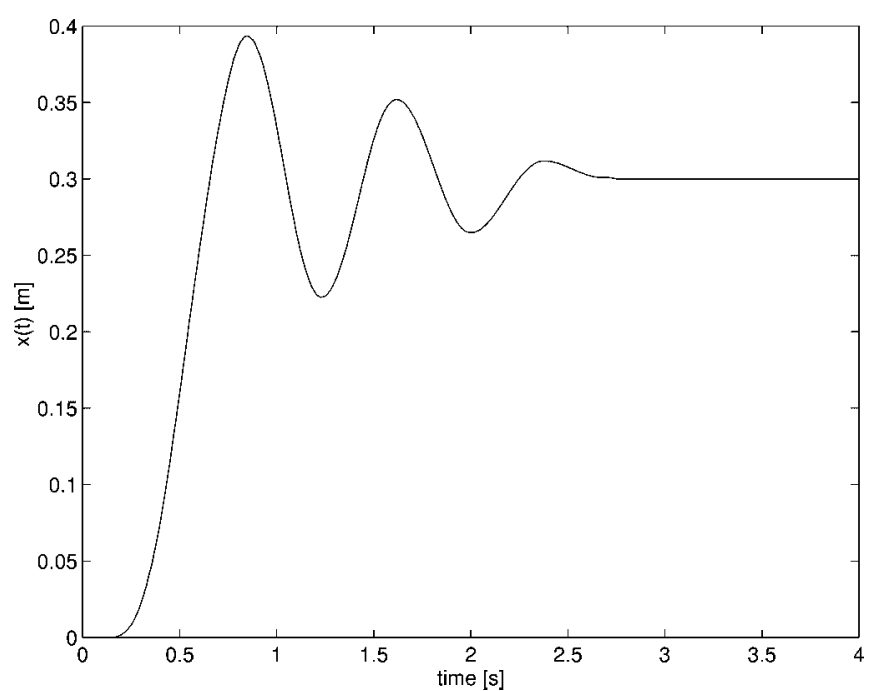

Fig. 20. Experimental motion of the load using a bang-acceleration function with motion time $\tau=0.75 \mathrm{~s}$ (bang-bang I case).

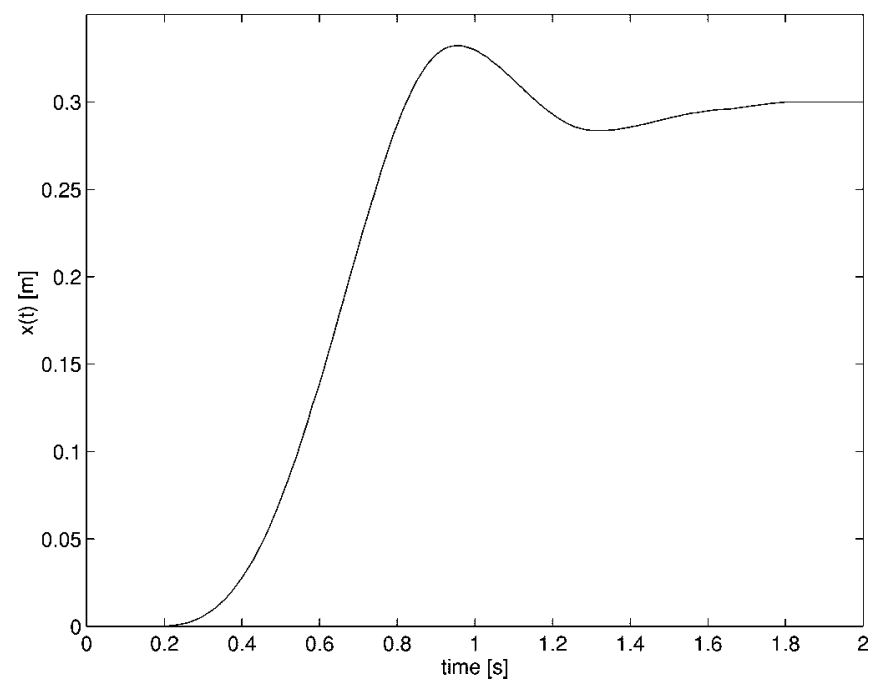

Fig. 21. Experimental motion of the load using a bang-bang acceleration function with motion time $\tau^{*}=1.11 \mathrm{~s}$ (bang-bang II case).

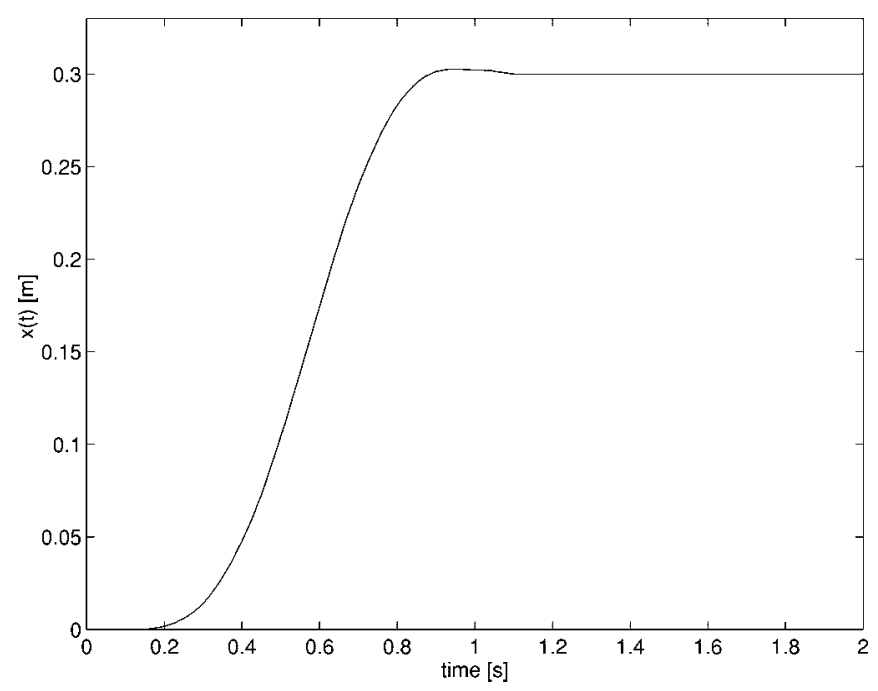

Fig. 22. Experimental motion of the load using the ZV impulse shaper method.

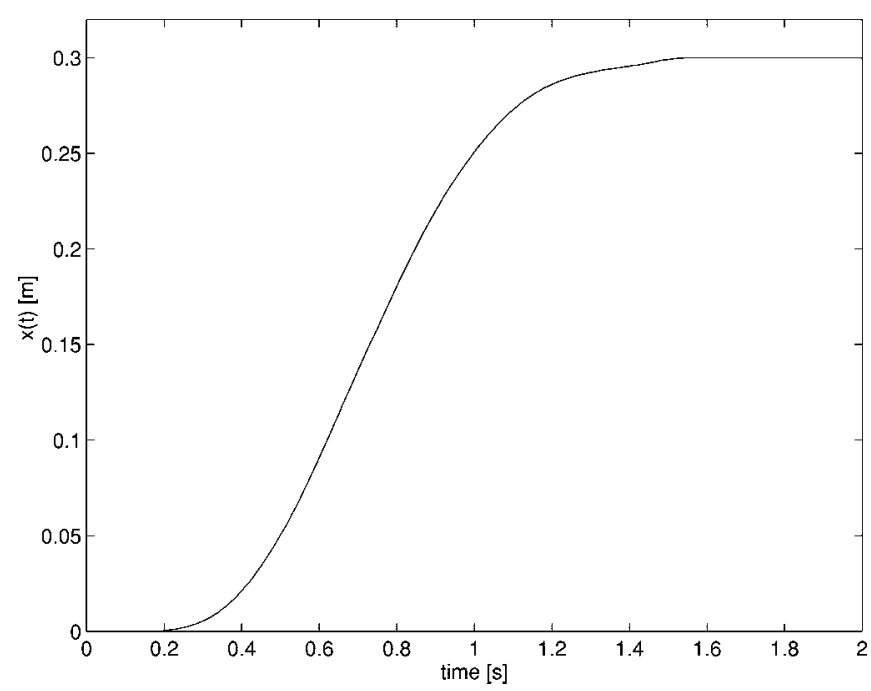

Fig. 23. Experimental motion of the load using the ZVD impulse shaper method.

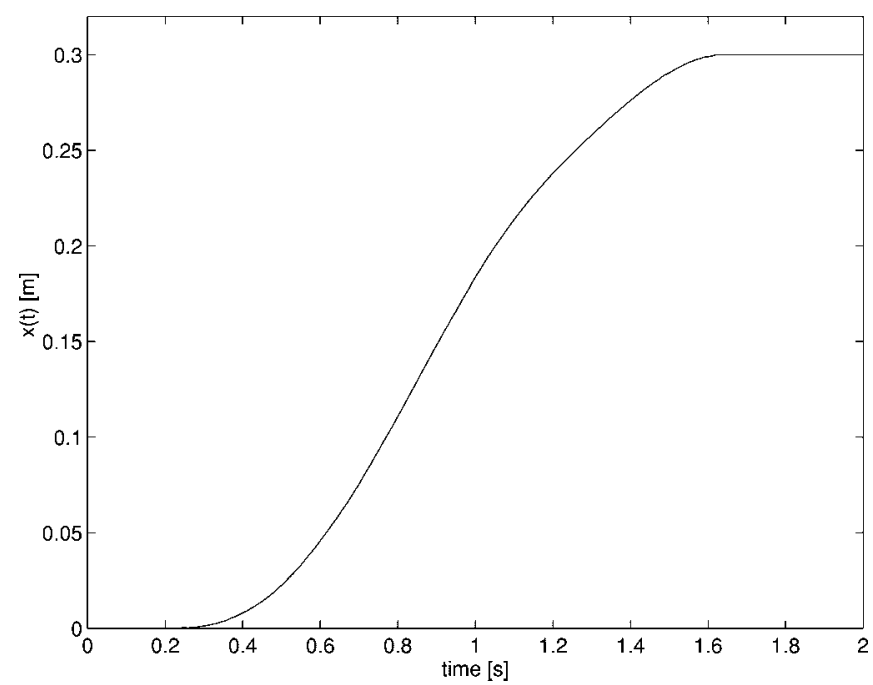

Fig. 24. Experimental motion of the load using the ZVDD impulse shaper method.

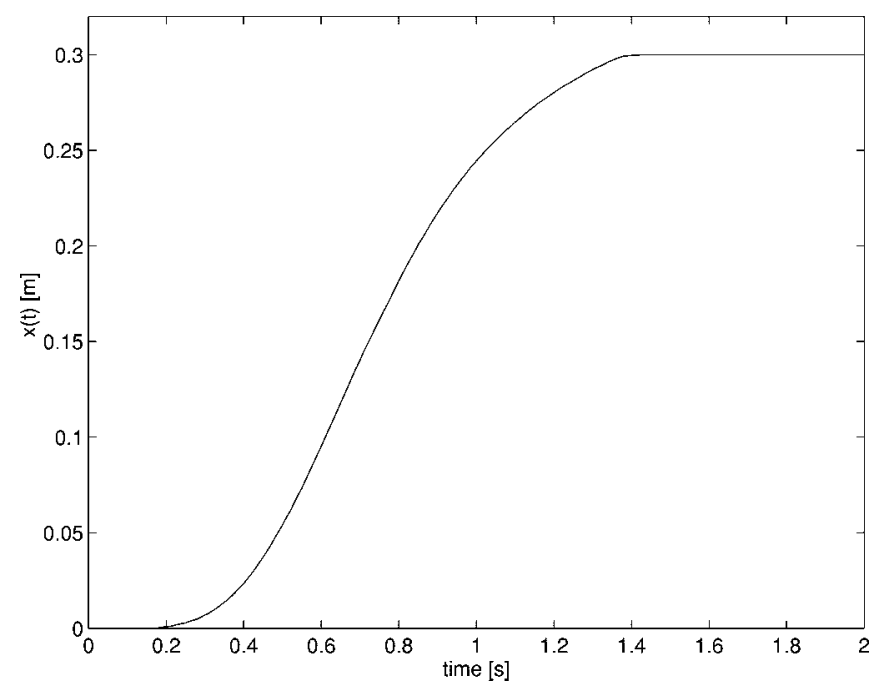

Fig. 25. Experimental motion of the load using the EI impulse shaper method. 


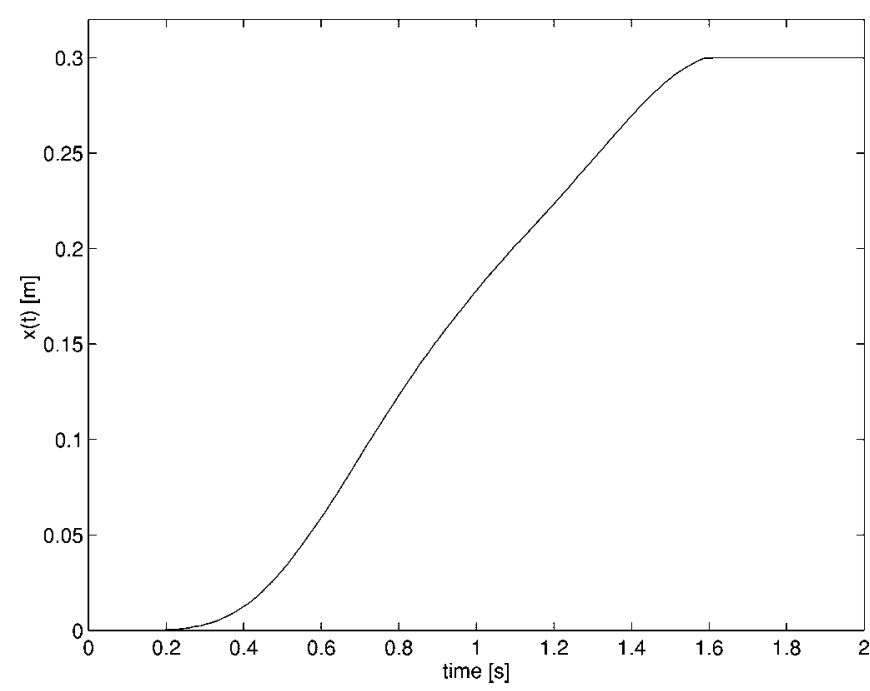

Fig. 26. Experimental motion of the load using the two-humped EI impulse shaper method.

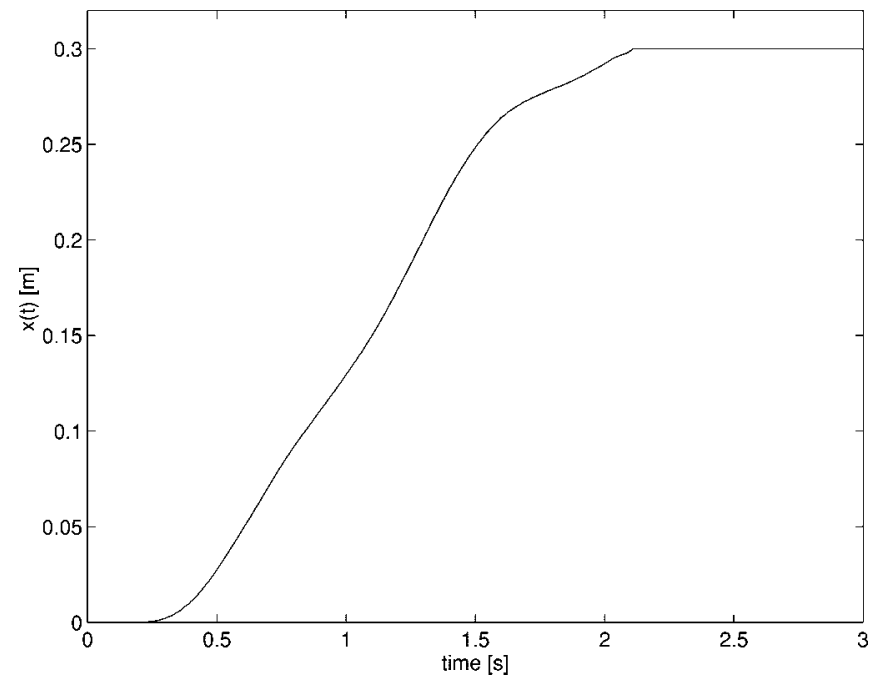

Fig. 27. Experimental motion of the load using the three-humped EI impulse shaper method.

TABLE II

EXPERIMENTAL COMPARISON OF THE DIFFERENT METHODOLOGIES

\begin{tabular}{l|c|c}
\hline Methodology & $\begin{array}{c}\text { Scheduled } \\
\text { motion time }[\mathrm{s}]\end{array}$ & $\begin{array}{c}\text { Maximum amplitude of } \\
\text { residual vibration }[\mathrm{cm}]\end{array}$ \\
\hline System inversion & 1.11 & 0 \\
\hline Bang-bang I & 0.75 & 9.34 \\
\hline Bang-bang II & 1.11 & 3.23 \\
\hline ZV shaper & 1.13 & 0.26 \\
\hline ZVD shaper & 1.50 & 0 \\
\hline ZVDD shaper & 1.88 & 0 \\
\hline EI shaper & 1.50 & 0 \\
\hline Two-humped EI shaper & 1.87 & 0 \\
\hline Three-humped EI shaper & 2.25 & 0 \\
\hline
\end{tabular}

whose duration time is chosen to be equal to $0.874 \mathrm{~s}$, the optimal motion time of the inversion-based approach (bang-bang II case). It results that the amplitude of the residual vibration in the nominal case is $9.9 \cdot 10^{-3} \mathrm{~m}$ (the motion of the load is plotted in Fig. 9) and in the worst case configuration of $k$ and $c$ is $25.6 \cdot 10^{-3} \mathrm{~m}$ (see Fig. 10).
Remark 2: It is worth stressing that, for the bang-bang cases (and for the input shaping techniques, see Section V), the input duration time, i.e., the time interval necessary for the input signal to complete the transition from 0 to $q$, is equal to the scheduled (load) motion time. On the contrary, for the system-inversion-based method, the actual input duration time can be longer than the scheduled motion time $\tau$ [see (13) and (14)]. However, for the presented numerical case, the input $y\left(t ; \tau^{*}\right) \simeq q=1$ for $t \geq \tau^{*}$ because it happens that $y\left(\tau^{*} ; \tau^{*}\right) \simeq q=1$. From a physical viewpoint, this means that, for $t=\tau^{*}$, the system has almost reached the equilibrium, so that the input duration time is equal to the optimal scheduled motion time $\tau^{*}$.

Remark 3: It is apparent from (14) and (16) that the computational complexity of the noncausal input is moderate. Indeed, the implementation of this input command can be easily done,even on a low-cost digital processor with the computation required in run time that is typically a small fraction of the sampling period.

\section{Comparison With the InPUT ShaPING TeChNiQues}

A comparison with the input shaping method has been executed in order to prove the effectiveness of the novel approach presented in this paper. We consider a bang-bang function for the acceleration input whose maximum amplitude is $y_{\max }^{(2)}=10$ $\mathrm{m} \cdot \mathrm{s}^{-2}$ as above (bang-bang I case). Then, the corresponding position reference signal has been convolved with different sequences of impulses (ZV, ZVD, ZVDD, EI, two-humped EI, and three-humped EI) [6], [9], [10]. A robustness analysis has been carried out as in the previous section. Results are shown in Figs. 11-16. The scheduled motion time, increased by the effect of the convolution of the input function, in the different cases are shown in Table I, where the main results regarding the simulation example are summarized. It appears that the most robust methods, i.e., those with minimal worst case residual vibration amplitude, are the ZVDD shaping and the two- and three-humped EI shaping. However, these methods have the longest motion times. Comparing the methods which do not have residual vibrations in the nominal case, the best one in terms of minimum motion time (equal to $0.745 \mathrm{~s}$ ) is the ZV shaping which has a relatively high worst case residual vibration amplitude (equal to $12.7 \mathrm{~mm}$ ). Pondering both the scheduled motion time and the robustness performance, it appears that the best methods are the system-inversion-based one and the ZVD shaping, with the latter performing slightly better than the former.

\section{EXPERIMENTAL RESULTS}

The system-inversion-based methodology has been tested experimentally on a testbed system, made by Quanser Consulting, composed of two carts, coupled by a spring, that slide on a ground stainless steel shaft (see Fig. 17). The first cart (motor cart) is driven by a dc motor, and it is equipped with a potentiometer to measure its position, while the second cart (load cart) only has a potentiometer. The overall control architecture has been implemented by means of a CPU Pentium $233 \mathrm{MHz}$ with an I/O board and a real-time environment which assures a 
control frequency of $200 \mathrm{~Hz}$. The aim of our experiments is to move the load cart from 0 to $q=0.3 \mathrm{~m}$, according to the predetermined polynomial output function of fifth order, by controlling the position (input function) of the motor cart, by means of a simple proportional plus integral plus derivative (PID) controller. The parameters of the systems have been estimated by means of a simple procedure, i.e., a force impulse has been applied to the load cart, keeping fixed the position of the motor cart. Evaluating the impulse response, the stiffness constant and the damping ratio (due to the friction) can be easily calculated. It results in $k=49.44 \mathrm{~kg} \cdot \mathrm{m}^{-2}$ and $c=0.412 \mathrm{~kg} \cdot \mathrm{m}^{-1}$, while the mass of the load cart is $m=0.713 \mathrm{~kg}$ (i.e., $\omega_{n}=8.327$ $\mathrm{rad} \cdot \mathrm{s}^{-1}$ and $\left.\xi=0.035\right)$. The minimum motion time $\tau^{*}$ has been determined by means of the algorithm described in Section III, taking into account the maximum voltage $(5 \mathrm{~V})$ that can be provided to the motor. It results in $\tau^{*}=1.11 \mathrm{~s}$. The theoretical output function and the experimentally obtained one are plotted in Fig. 18, while the value of motor voltage during the motion is plotted in Fig. 19. It appears that no vibrations occur during the motion, as expected, and that the value of $5 \mathrm{~V}$ for the motor voltage is attained, but not exceeded. It should be noted that the slight difference between the theoretical and the actual load motions is due to the presence of unmodeled nonlinearities (e.g., friction effects).

The use of a bang-bang acceleration input has also been tested experimentally. The minimum scheduled motion time allowed by the system is, in this case, equal to $0.75 \mathrm{~s}$, which leads to the load motion shown in Fig. 20 (bang-bang I case). In addition, a bang-bang acceleration profile of duration time equal to $1.11 \mathrm{~s}$ has also been adopted (bang-bang II case). The obtained load motion is plotted in Fig. 21. In both cases, it turns out that the residual vibration is much higher than in the system-inversion-based approach, so that the latter methodology is more convenient.

In order to compare again the proposed methodology with the input shaping one, the different input shapers have been also experimentally tested. Plots of the corresponding load motions are in Figs. 22-27.

Experimental results are summarized in Table II. It can be seen that, for all the methodologies tested experimentally, with the exception of the bang-bang ones and the $\mathrm{ZV}$ shaper, no residual vibration occurs at the end of the motion. Hence, it results that the technique proposed in this paper is the most effective in the experiments, since it has the minimum transition time among the methods that do not exhibit residual vibration.

\section{CONCLUSIONS}

A novel methodology for the open-loop control of high-performances servosystems with elastic transmission has been presented. It is based on an appropriate choice of the motion law and on a noncausal system inversion which guarantees that actuator constraints are fully satisfied. The approach appears to be very effective in reducing the residual vibration, and the smoothness of the control input makes it inherently robust to modeling errors, especially in case the stiffness constant is greater than expected (i.e., the system is more rigid). It also has to be stressed that robustness increases as long as the motion time increases This provides a useful flexibility in the motion planning phase, since, taking into account actuator limits, time intervals of the automated cell where the system is situated can be usefully exploited in order to increase robustness. Moreover, limits on the derivative of an arbitrary order of the input can be assigned in order to enhance smoothness so that actuator dynamics is taken into account. It has been shown that no particular problems (e.g., due to the computational complexity) arise in the practical implementation of the methodology, and the flexibility and readiness of the design makes it an attractive alternative to the traditional input shaping techniques.

\section{REFERENCES}

[1] T. Totani and H. Nishimura, "Final-state control using compensation input," Trans. Soc. Instrum. Contr. Eng., vol. 30, no. 3, pp. 253-260, 1994.

[2] F. L. Lewis, Optimal Control. New York: Wiley, 1986.

[3] D. M. Aspinwall, "Acceleration profiles for minimizing residual response," ASME J. Dynam. Syst., Meas., Contr., vol. 102, pp. 3-6, Mar. 1980.

[4] P. Meckl and W. Seering, "Active damping in a three-axis robotic manipulator," ASME J. Vibr., Acoust., Stress, Reliab. Des., vol. 107, pp. 38-46, Jan. 1985.

[5] — "Minimizing residual vibration for point-to-point motion," ASME J. Vibr., Acoust., Stress, Reliab. Des., vol. 107, pp. 378-382, Oct. 1985.

[6] N. C. Singer and W. P. Seering, "Preshaping command inputs to reduce system vibration," ASME J. Dynam. Syst., Meas., Contr., vol. 112, pp. 76-82, Mar. 1990.

[7] J. M. Hyde and W. P. Seering, "Using input command pre-shaping to suppress multiple mode vibration," in Proc. IEEE Int. Conf. Robotics and Automation, vol. 3, Sacramento, CA, 1991, pp. 2604-2609.

[8] T. Singh and G. R. Heppler, "Shaped input control of a system with multiple modes," ASME J. Dynam. Syst., Meas., Contr., vol. 115, pp. 341-347, Sept. 1993.

[9] W. Singhose, W. Seering, and N. Singer, "Residual vibration reduction using vector diagrams to generate shaped inputs," ASME J. Mech. Des., vol. 116, pp. 654-659, June 1994.

[10] W. E. Singhose, L. J. Porter, T. D. Tuttle, and N. C. Singer, "Vibration reduction using multi-hump input shapers," ASME J. Dynam. Syst., Meas., Contr., vol. 119, pp. 320-326, June 1997.

[11] W. Singhose, E. Crain, and W. Seering, "Convolved and simultaneous two-mode input shapers," Proc. Inst. Elect. Eng.-Contr. Theory Applicat., vol. 144, no. 6, pp. 515-520, Nov. 1997.

[12] W. A. Porter, "An algorithm for inverting linear dynamic systems," IEEE Trans. Automat. Contr., vol. AC-14, pp. 702-704, Dec. 1969.

[13] L. M. Silverman, "Inversion of multivariable linear systems," IEEE Trans. Automat. Contr., vol. AC-14, pp. 270-276, June 1969.

[14] P. J. Moylan, "Stable inversion of linear systems," IEEE Trans. Automat. Contr., vol. AC-22, pp. 74-78, Feb. 1977.

[15] E. Soraka and U. Shaked, "On the geometry of the inverse system," IEEE Trans. Automat. Contr., vol. AC-31, pp. 751-754, Aug. 1986.

[16] R. W. Brockett and M. D. Mesarovic, "The reproducibility of multivariable control systems," J. Math. Anal. Appl., vol. 11, pp. 548-563, July 1965.

[17] G. Basile and G. Marro, "On the perfect output controllability of linear dynamic system," Ric. Autom., vol. 2, no. 1, pp. 1-10, 1971.

[18] L. R. Hunt, G. Meyer, and R. Su, "Noncausal inverses for linear systems," IEEE Trans. Automat. Contr., vol. 41, pp. 608-611, Apr. 1996.

[19] S. Devasia and B. Paden, "Exact output tracking for nonlinear timevarying systems," in Proc. IEEE Int. Conf. Decision and Control, Lake Buena Vista, CA, 1994, pp. 2346-2355.

[20] S. Devasia, B. Paden, and C. Rossi, "Minimal transient regulation of flexible structures," in Proc. IEEE IECON'94, vol. 3, Bologna, Italy, 1994, pp. 1761-1766.

[21] G. Marro and A. Piazzi, "A geometric approach to multivariable perfect tracking," in Proc. 13th IFAC World Congr., vol. C, San Francisco, CA, 1996, pp. 241-246.

[22] G. Legnani and R. Faglia, "Harmonic drive transmission: The effects of their elasticity, clearance and irregularity on the dynamic behavior of an actual SCARA robot," Robotica, vol. 10, no. 4, pp. 369-375, 1992. 
[23] R. Adamini, R. Faglia, and G. Incerti, "Parametric motion planning techniques for servosystems endowed with elastic transmission," Mach. Vibr., vol. 4, no. 1, pp. 14-21, 1995.

[24] H. Ratschek and J. Rokne, New Computer Methods for Global Optimization. Chichester, U.K.: Ellis Harwood, 1988.

[25] A. Piazzi and A. Visioli, "An interval algorithm for minimum-jerk trajectory planning of robot manipulators," in Proc. 36th IEEE Int. Conf. Decision and Control, vol. 2, San Diego, CA, 1997, pp. 1924-1927.

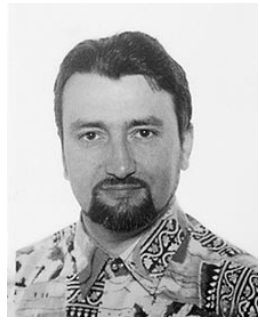

Aurelio Piazzi (M'92) received the Laurea degree in nuclear engineering and the Ph.D. degree in system engineering from the University of Bologna, Bologna, Italy, in 1982 and 1987, respectively.

From 1990 to 1992, he was a Research Associate in System Theory, D.E.I.S., University of Bologna. Since November 1992, he has been an Associate Professor of Automatic Control, Dipartimento di Ingegneria dell' Informazione, University of Parma, Parma, Italy. His main research interests are in system and control theory and related engineering applications. His current research activities focus on noncausal regulation and on methods of global optimization applied to the analysis and design of robust control systems.

Dr. Piazzi is a member of the International Federation for Automatic Control and Society for Industrial and Applied Mathematics.

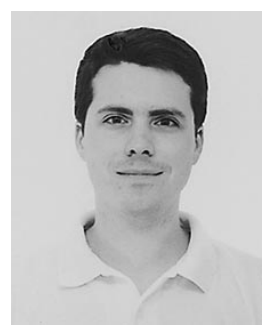

Antonio Visioli (S'96-M'00) received the Laurea degree in electronic engineering from the University of Parma, Parma, Italy, and the Ph.D. degree in applied mechanics from the University of Brescia, Brescia, Italy, in 1995 and 1999, respectively.

$\mathrm{He}$ is currently an Assistant Professor of Automatic Control, Department of Electronics for Automation, University of Brescia. His research interests include industrial robot control and trajectory planning, systems-inversion-based control, and PID control. 